\title{
Ezrin expression is related to poor prognosis in FIGO stage I endometrioid carcinomas
}

\author{
Martin Köbel ${ }^{1}$, Tina Langhammer ${ }^{1}$, Stefan Hüttelmaier ${ }^{3}$, Wolfgang D Schmitt ${ }^{1}$, \\ Karen Kriese $^{1}$, Jürgen Dittmer ${ }^{2}$, Hans-Georg Strauss ${ }^{2}$, Christoph Thomssen ${ }^{2}$ \\ and Steffen Hauptmann ${ }^{1}$ \\ ${ }^{1}$ Institute of Pathology, Martin-Luther-University Halle-Wittenberg, Halle, Germany; ${ }^{2}$ Department of \\ Gynecology, Martin-Luther-University Halle-Wittenberg, Halle, Germany and ${ }^{3}$ ZAMED, Department of \\ Medicine, Martin-Luther-University Halle-Wittenberg, Halle, Germany
}

\begin{abstract}
As a cortical cytoskeletal protein, ezrin adapts the cytoplasmic tail of CD44 to the actin-based cytoskeleton and is functionally involved in migration and adhesion that are prerequisites for metastasis. To assess the importance of ezrin and its associated protein osteopontin for the progression of endometrioid carcinoma in FIGO stage I, we analyzed paraffin-embedded tissue from 164 patients by immunohistochemistry and correlated these data with clinicopathological parameters. Ezrin was expressed in normal proliferating endometrial glands, as was confirmed by quantitative PCR and immunohistochemistry. In endometrioid carcinoma, enhanced ezrin expression correlated with a reduced overall survival in univariate analysis $(P=0.041)$. In contrast, no significant correlation was found for osteopontin. In multivariate survival analysis, among FIGO grade 3 and age, ezrin was still found to be an independent risk factor (relative risk 2.2, confidence interval 1.0-5.4, $P=0.047$ ). Hence, elevated ezrin expression is a new independent prognostic marker in FIGO stage I endometrioid carcinoma, and thus provides further evidence for an important role of ezrin in tumor progression.

Modern Pathology (2006) 19, 581-587. doi:10.1038/modpathol.3800567
\end{abstract}

Keywords: ezrin; osteopontin; expression; endometrioid carcinoma; FIGO I; prognosis

Endometrial carcinoma was responsible for an estimated number of 40880 cases and 7310 deaths in the United States in 2005. ${ }^{1}$ In Germany, approximately 11250 new cases of endometrial cancer and 2536 cancer-related deaths were reported in $2003{ }^{2}$ With advances in surgical treatment, disease-specific mortality declined over the past 30 years. However, diagnosis of endometrial carcinoma still imposes a substantial burden on patients. Prognosis is dependent on classical clinicopathological parameters like FIGO stage and grade as well as histological type (endometrioid vs nonendometrioid carcinomas). Nonetheless, even within a group of patients with a low risk of disease progression (endometrioid type, FIGO stage I) some patients suffer from early recurrence or disease-related death. Therefore, additional molecular factors seem to be needed to individualize both patient prognosis and

Correspondence: Dr M Köbel, MD, Institute of Pathology, MartinLuther-University Halle-Wittenberg, Magdeburger Str. 14, Halle D-06097, Germany.

E-mail: martin.koebel@medizin.uni-halle.de

Received 08 December 2005; revised and accepted 10 January 2006 therapy. Because cell migration is an essential step in tumor progression, we have investigated the expression of two proteins involved in the regulation of cell motility. Ezrin, as a member of the ERMfamily (ezrin, radixin, moesin), adapts CD44 to actin-based cytoskeleton, and therefore organizes the status of the membrane-cytoskeleton interactions. ${ }^{3}$ Recent studies suggest a metastasis-promoting role for ezrin in several tumor entities by facilitating tumor cell motility. These include pancreatic carcinoma, ${ }^{4}$ breast carcinoma, ${ }^{5}$ ovarian carcinoma, ${ }^{6,7}$ melanoma, ${ }^{8}$ and osteosarcoma. ${ }^{9}$ Furthermore, inhibition of ezrin by antisense oligonucleotides blocked invasiveness of endometrial adenocarcinoma cell lines. ${ }^{7}$ However, in endometrial carcinoma, only one study demonstrated an overexpression of ezrin in carcinoma compared to simple hyperplasia or normal endometrium, although its prognostic significance was not evaluated. ${ }^{10}$

Recently, other proteins that associate with ezrin and CD44 have been described. One of these is osteopontin, a mineral binding glycoprotein that under physiological conditions is secreted to the extracellular matrix by osteoblasts to provide an 
anchor to the bone matrix via $\alpha_{\mathrm{v}} \beta 3$ integrin. At the cytoplasmic face of cell adhesion sites osteopontin is able to form a submembranal complex with ezrin and CD44. ${ }^{11}$ Similar to ezrin, osteopontin seems to be involved in cell adhesion and migration. ${ }^{12}$ Its expression has been investigated in a number of human tumors. ${ }^{13}$ In gynecological tumors including breast and ovarian carcinomas, the overexpression of intracellular osteopontin was associated with poor prognosis. ${ }^{14,15}$

In order to gain further insights into the importance of ezrin and osteopontin for progression of endometrial cancer, we correlated the expression of both proteins with clinicopathological parameters as well as patients survival in a large set of FIGO stage I endometrioid carcinomas comprising 164 samples.

\section{Materials and methods}

\section{Quantitative Polymerase Chain reaction}

Tissue of seven endometrioid carcinomas and associated tumor-free endometrium was dissected from a pathologist during frozen section analysis, immediately quick-frozen and stored at $-80^{\circ} \mathrm{C}$ until analysis. Before homogenizing, tissue samples were confirmed for tumor content by H\&E section. Total RNA was extracted by RNeasy Kit (Qiagen) and reverse transcribed by MMLV-point mutant reverse transcriptase (Promega). SYBR Green quantitative PCR for ezrin (sense: $5^{\prime}$-gttttccccagttgtaatagtgcc- $3^{\prime}$, reverse: $5^{\prime}$-tccgtaattcaatcagtcctgc- $3^{\prime}$ ) or cyclophilin A (sense: $5^{\prime}$-gtcaacccaccgtgtctt- ${ }^{\prime}$, reverse: $5^{\prime}$ ctgctgtctttgggaccttgt- $3^{\prime}$ ) was performed using a ROX-supplemented $2 \times$ Taq Mix (Promega). The relative amount of RNA was calculated based on the $\Delta C_{\mathrm{T}}$-method as described recently (Livak and Schmittgen, 2001). Cyclophilin A was used as an internal standard.

\section{Study Population}

Paraffin-embedded tissue samples from a total of 164 patients with FIGO stage I endometrioid carcinoma, which were diagnosed at the Institute of Pathology, Martin-Luther-University Halle-Wittenberg, Halle, Germany, between 1998 and 2002 were used for this study. Furthermore, five proliferative and two secretory endometrium samples were examined. The study was approved by local ethical committee. Patient's age and FIGO stage were retrieved from clinical and pathological files (Table 1). All histological slides were re-evaluated by two pathologists ( $\mathrm{MK} \& \mathrm{SH}$ ). Histology was classified according to WHO and grading was assessed according to the FIGO system. Maximal primary surgery was given if patients with pT1aG1/ G2 or pT1bG1 tumors had an abdominal hysterectomy and bilateral salpingo-oophorectomy. Tumors with pT1bG2 or pT1c were only expected to be treated maximal if sampling of lymph nodes was carried out. Thus, $118(72 \%)$ of patients has a maximal primary surgery. Follow-up for this cohort was updated to October 2005 by inquiry from the tumor and local population registry. Complete data with a median follow-up time were available for 164 $(100 \%)$ patients with a median follow-up time of 57.4 months (range 0.13-93.4 months). The median age was 68.9 years. A total of $27(16.5 \%)$ patients died of disease. Four $(2.4 \%)$ patients died of other

Table 1 Correlation of clinicopathological parameters of 164 patients with ezrin and osteopontin expression

\begin{tabular}{|c|c|c|c|c|c|c|c|c|}
\hline Characteristic & Ezrin weak & Ezrin strong & All cases & P-value & Osteopontin weak & Osteopontin strong & All cases & $\mathrm{P}$-value \\
\hline All cases & 81 & 83 & 164 & & 84 & 80 & 164 & \\
\hline \multicolumn{9}{|c|}{ Age at diagnosis } \\
\hline$\leq 68.9$ years & 42 & 40 & 82 & 0.63 & 39 & 43 & 82 & 0.34 \\
\hline$>68.9$ years & 39 & 43 & 82 & & 45 & 37 & 82 & \\
\hline \multicolumn{9}{|l|}{$p T$} \\
\hline $1 a$ & 23 & 7 & 30 & 0.03 & 16 & 14 & 30 & 0.36 \\
\hline $1 \mathrm{~b}$ & 43 & 51 & 94 & & 44 & 50 & 94 & \\
\hline $1 \mathrm{c}$ & 15 & 25 & 40 & & 24 & 16 & 40 & \\
\hline \multicolumn{9}{|c|}{ Maximal surgery } \\
\hline Yes & 62 & 56 & 118 & 0.19 & 58 & 60 & 118 & 0.39 \\
\hline No & 19 & 27 & 46 & & 26 & 20 & 46 & \\
\hline \multicolumn{9}{|l|}{ FIGO grade } \\
\hline 1 & 62 & 64 & 126 & 0.33 & 65 & 61 & 126 & 0.75 \\
\hline 2 & 16 & 12 & 28 & & 15 & 13 & 28 & \\
\hline 3 & 3 & 7 & 10 & & 4 & 6 & 10 & \\
\hline \multicolumn{9}{|c|}{ Osteopontin expression } \\
\hline Weak & 44 & 40 & 84 & 0.43 & & & & \\
\hline Strong & 37 & 43 & 80 & & & & & \\
\hline
\end{tabular}


cause and were censored for survival analysis, 5-year survival rate was $82.6 \%$.

\section{Immunohistochemistry}

Immunohistochemistry was performed using standard procedures as recently described. ${ }^{6}$ For detection of ezrin and osteopontin we used mouse monoclonal antibodies clone 3C12 from Sigma (Deisenhofen, Germany) and clone OP3N from Novocastra (Newcastle, UK). To retrieve antigens, slides were treated with sodium citrate buffer, $\mathrm{pH} 6$, in a microwave oven for $4 \times 5 \mathrm{~min}$. Primary antibodies were diluted 1:5.000 for ezrin and 1:50 for osteopontin in PBS and incubated at $37^{\circ} \mathrm{C}$ for $30 \mathrm{~min}$. After washing with PBS, a biotinylated secondary antibody followed by enhancing streptavidin-horseradish peroxidase was applied. Red color was developed by aminoethylcarbazol (AEC, Zytomed, Berlin, Germany). To minimize daily variation, the slides were immunostained together in three runs. As positive control for ezrin, colonic mucosa showed a faint apical staining and additional expression in scattered lymphocytes. Predecidualized endometrial stroma cells served as positive control for osteopontin.

\section{Evaluation of Immunohistochemical Staining}

Tumor staining was independently examined by two investigators (MK and TL) blinded to the clinical data. Each tumor was represented by one tissue slide. Since staining intensity did not differ significantly, percentage of positive tumor cells was estimated. In cases where the score differed more than $5 \%$, consensus was achieved at a multiheaded microscope. Moreover, the subcellular localization of staining (predominantly cytoplasmic, pure apical, or mixed) as well as the stromal staining of osteopontin was recorded.

\section{Statistical Analysis}

For statistical analysis, the cutoff level for grouping in weak or strong expression was defined at the median percentage score that was $15 \%$ of tumor cells for ezrin and $10 \%$ of tumor cells for osteopontin. Statistical correlation between clinicopathological factors and expression of ezrin and osteopontin was assessed by using $\chi^{2}$ test for trends. The probability of differences in overall survival as a function of time was determined by Kaplan-Meier test, with probing for significance by a log-rank test. Multivariate regression analysis based on the Cox's proportional hazard model was used to test the independence of these parameters in the prediction of overall survival. Generally, $P$-values smaller than 0.05 were considered to show a significant difference. For all statistical procedures SPSS v12.0 software (SPSS GmbH, Munich, Germany) was used.

\section{Results}

\section{Ezrin Protein Expression in Endometrial Tissue}

Immunoreactivity of ezrin was detected in all phases of normal cycling endometrium. As shown in Figure 1a, proliferative endometrium showed an apical membranous staining with an additional faint cytoplasmic signal that was pronounced at the cell basis.

In endometrioid carcinomas, ezrin was at least focally expressed in $93 \%$ of cases. Only $7 \%$ of cases were completely negative. Expression was quite heterogeneous with a wide range from only a few to nearly $100 \%$ of positive tumors cells. The cutoff value for weak and strong expressing cases was specified at the median percentage score of positive tumor cells resulting in a value of $15 \%$. The cellular expression pattern in carcinomas was predominantly cytoplasmic (Figure 1b-d). Interestingly, tumor cells with an intensive cytoplasmic staining seemed to loose their cell-to-cell contacts. This was either observed in free floating cell groups that were detached from papillae (Figure 1c) or in cells at the tumor-host interface (Figure 1c and d). Except for scattered lymphocytes, tumor stroma was always negative for ezrin staining.

\section{Ezrin mRNA Expression in Endometrial Tissue}

To investigate expression of ezrin mRNA, we analyzed seven endometrioid carcinomas as well as associated tissue free of tumor by quantitative PCR. For each sample, ezrin expression level was compared between normal (100\%) and tumor tissue. Additionally, we controlled tumor samples for the ratio of epithelial vs stromal component by verifying a corresponding $\mathrm{H} \& \mathrm{E}$ section. Differences in the tumor/stroma ratio were adjusted to $100 \%$. Two of the analyzed endometrioid carcinomas showed a decrease of ezrin mRNA, while a slight increase of ezrin expression was observed in three cases and an up to four-fold overexpression in two cases (Figure 2). This suggests that endometrioid carcinomas can be divided in two groups: one with increased and another with retained or reduced ezrin mRNA concentrations.

\section{Osteopontin Expression in Endometrial Tissue}

Osteopontin is also expressed in normal cycling endometrium. Proliferating and secretory endometrium showed a diffuse cytoplasmic signal in epithelial cells (Figure 1e). Predecidualized stroma cells served as internal control showing strong cytoplasmic expression whereas other stromal components were negative. 

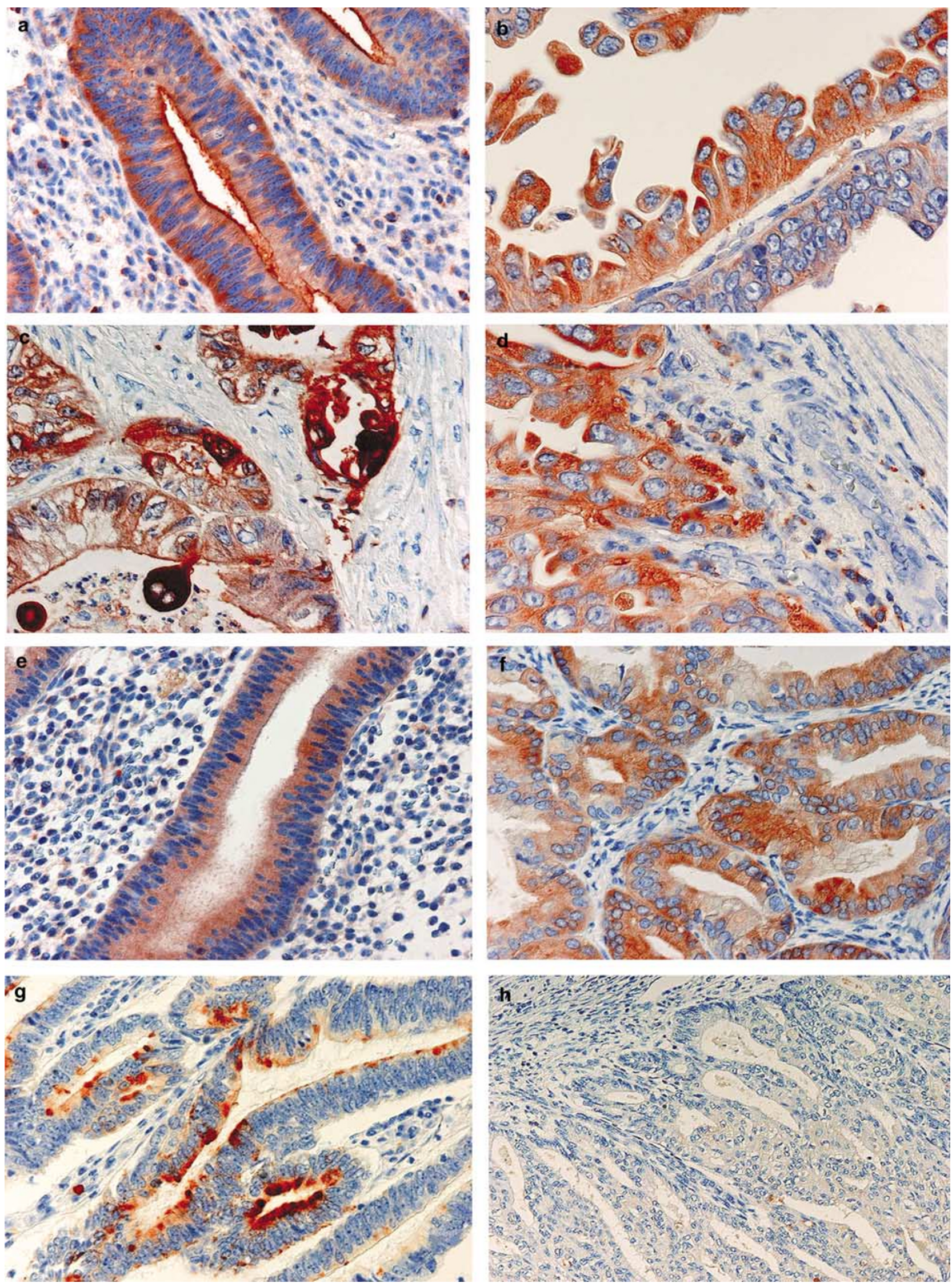
Most of endometrioid carcinomas expressed osteopontin whereas $29 \%$ of cases were completely negative (Figure 1h). The expression was also heterogeneous. The median percentage score to separate strong and weak expressing cases was set to $10 \%$ of tumor cells positive for osteopontin. The cellular localization in endometrioid carcinomas was either a diffuse or patchy cytoplasmic staining (Figure 1f and g). Stromal osteopontin was of negligible amounts.

\section{Ezrin and Osteopontin Expression in FIGO I Endometrioid Carcinomas: Univariate Correlation with Overall Survival}

In univariate survival analysis, a significant correlation of strong ezrin expression with overall survival was found (Figure $3, P=0.041$ ). Strong ezrin expression was also correlated with pT stage. However, no correlation was observed between ezrin expression and age, FIGO grade, maximal surgery, or osteopontin expression (Table 1). Tha 5-year survival rate was decreased for patients with strong ezrin-expressing tumors from 89 to $76 \%$ (Table 2). Other clinicopathological parameters, such as age, pT stage, maximal surgery and FIGO grade were also significantly associated with overall survival (Table 2). In contrast, patients with strong osteopontin expression had a more favorable outcome (Figure 3), even though this difference was not significant.

\section{Ezrin Expression in Endometrial Carcinomas: Multivariate Correlation with Overall Survival}

For multivariate analysis, all factors with significance in univariate analysis were included in the

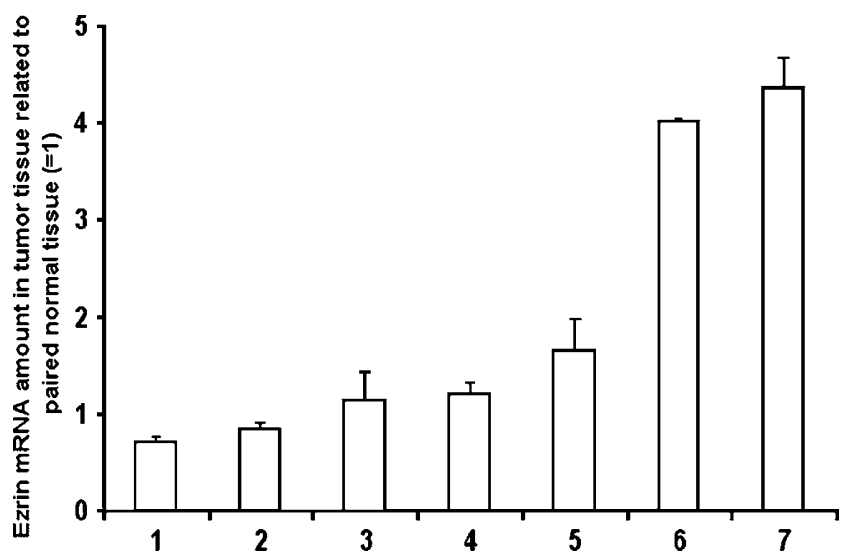

Figure 2 Expression of ezrin mRNA in seven endometrioid carcinomas. Each sample was normalized to cyclophylin A, to the relative tumor/stroma ratio of the sample and to tumor-free associated endometrium in percent. multivariate analysis using the Cox's hazard regression model. Multivariate risk factors in decreasing order were FIGO grade 3 , age $\geq 68.9$ and strong ezrin expression (Table 3 ).

\section{Discussion}

Positioned at the submembranal-cytoskeletal interface, ezrin may be a nexus in metastatic progression with a central role in the regulation of invasion. Ezrin is physiologically expressed in a variety of epithelial tissue including intestine, lung, and kidney. Within these tissues it is found in the apical region of microvilli presenting cells suggesting an involvement in defining cell polarity. However, besides its localization to microvilli, ezrin is also
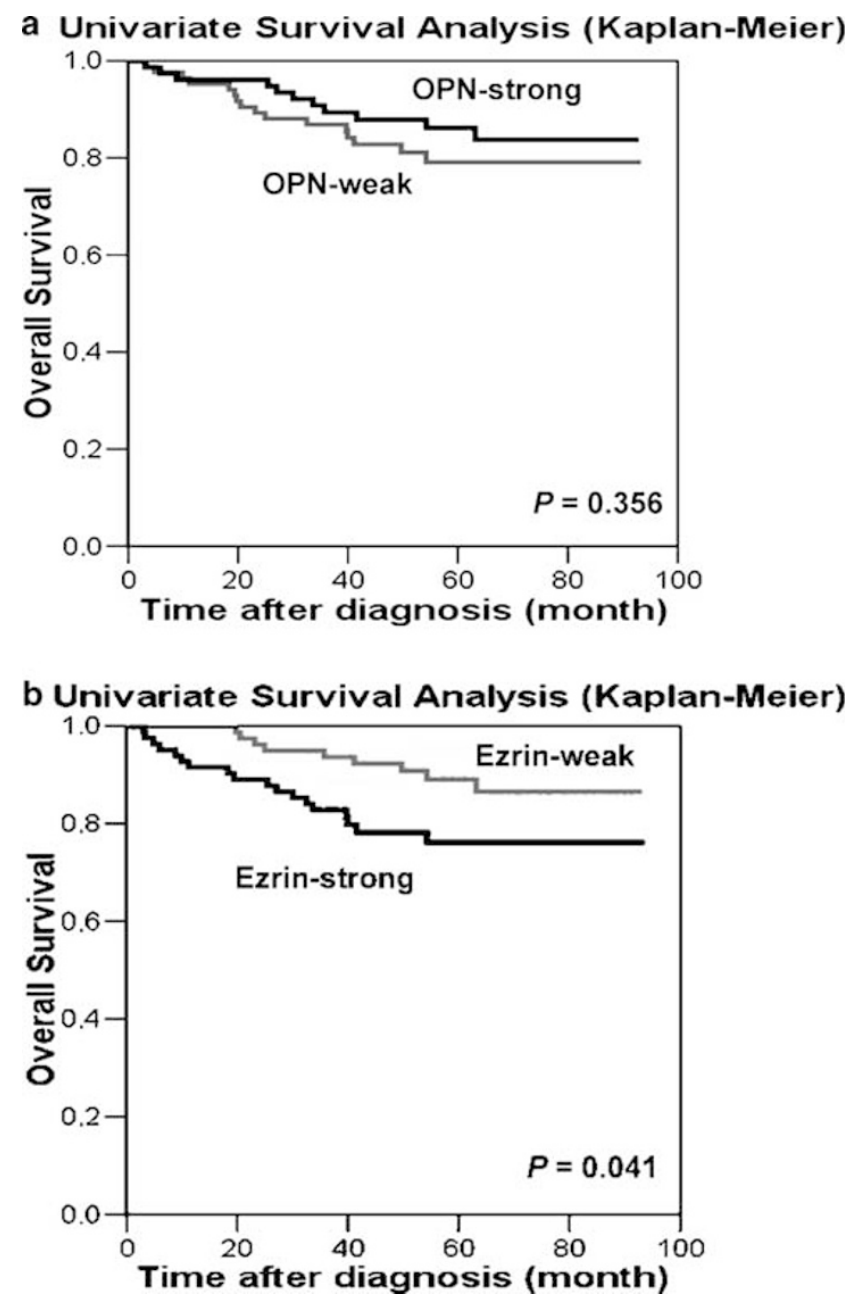

Figure 3 Univariate survival analysis (Kaplan-Meier). (a) Correlation of weak and strong ezrin expression with overall survival. (b) Correlation of weak and strong osteopontin expression with overall survival.

Figure 1 Ezrin (left) and osteopontin (right) expression by immunohistochemistry. (a) Proliferative endometrium with ezrin expression. (b-d) Endometrioid carcinoma with strong ezrin expression. (c and d) Note: cells with intense cytoplasmic ezrin expression are either detached or located at the tumor-host interface. (e) Proliferative endometrium with osteopontin expression. (f and g) Endometrioid carcinoma with strong osteopontin expression. (h) Endometrioid carcinoma negative for osteopontin. 
Table 2 Univariate survival analysis (Kaplan-Meier): 5-year survival rate of 164 patients with FIGO I endometrioid carcinomas according to clinicopathological factors and ezrin or osteopontin expression

\begin{tabular}{|c|c|c|c|c|c|}
\hline & Cases & Events & $\begin{array}{c}5 \text { year } \\
\text { survival } \\
\text { ratein \% }\end{array}$ & $\begin{array}{l}\text { Standard } \\
\text { error }\end{array}$ & $\begin{array}{c}\text { Log-rank } \\
\text { test (P- } \\
\text { value) }\end{array}$ \\
\hline All cases & 164 & 27 & 83 & 4 & \\
\hline \multicolumn{6}{|c|}{ Age at diagnosis } \\
\hline$\leq 68.9$ years & 82 & 6 & 92 & 3 & \multirow[t]{2}{*}{0.0017} \\
\hline$>68.9$ years & 82 & 21 & 73 & 5 & \\
\hline \multicolumn{6}{|l|}{$p T$} \\
\hline $1 a$ & 30 & & 100 & & \multirow[t]{3}{*}{0.0015} \\
\hline $1 \mathrm{~b}$ & 94 & 15 & 83 & 4 & \\
\hline 1c & 40 & 12 & 68 & 8 & \\
\hline \multicolumn{6}{|c|}{ Maximal surgery } \\
\hline Yes & 118 & 14 & 88 & 3 & \multirow{2}{*}{0.0047} \\
\hline No & 46 & 13 & 66 & 8 & \\
\hline \multicolumn{6}{|l|}{ FIGO grade } \\
\hline Grade 1 & 126 & 14 & 81 & 3 & \multirow[t]{3}{*}{$<0.0001$} \\
\hline Grade 2 & 28 & 7 & 71 & 10 & \\
\hline Grade 3 & 10 & 6 & 33 & 16 & \\
\hline \multicolumn{6}{|c|}{ Ezrin expression } \\
\hline Weak & 81 & 9 & 89 & 4 & \multirow[t]{2}{*}{0.041} \\
\hline Strong & 83 & 18 & 76 & 5 & \\
\hline \multicolumn{6}{|c|}{ Osteopontin expression } \\
\hline Weak & 84 & 16 & 79 & 5 & \multirow[t]{2}{*}{0.356} \\
\hline Strong & 80 & 11 & 86 & 4 & \\
\hline
\end{tabular}

Table 3 Multivariate survival analysis (Cox's regression model) for overall survival of 164 patients with FIGO I endometrioid carcinomas

\begin{tabular}{lccr}
\hline & Relative risk & $\begin{array}{c}\text { 95\% confidence } \\
\text { interval }\end{array}$ & P-value \\
\hline $\begin{array}{l}\text { FIGO grade } \\
\text { G1 }\end{array}$ & 1.00 & & \\
G2 & 2.34 & $0.87-6.25$ & 0.090 \\
G3 & 6.93 & $2.35-20.37$ & $<0.001$ \\
Age & & & \\
$\quad \leq 68.9$ years & 1.00 & & \\
$\quad>68.9$ years & 3.28 & $1.29-8.44$ & 0.013 \\
Ezrin expression & & & \\
$\quad$ Weak & 1.00 & & \\
Strong & 2.22 & $1.00-5.37$ & 0.047 \\
pT & & & \\
1a, 1b & 1.00 & & \\
1c & 1.50 & $0.55-4.10$ & \\
Maximal surgery & & & 0.423 \\
$\quad$ Yes & 1.00 & & \\
No & 1.03 & $0.36-2.92$ & \\
\hline
\end{tabular}

targeted to the leading edge of spreading cells suggesting an essential role in controlling cell motility. To date, several binding partners for ezrin have been identified. The N-terminal binding domain of ezrin mediates membrane attachment by binding the cytoplasmic tail of CD44, CD43, or intercellular adhesion molecules. On the other end, ezrin via its C-terminal domain associates with Factin and contributes to microfilament organization.

Recently, by use of similar methods, we analyzed ezrin expression in ovarian carcinomas and found a strong correlation with poor prognosis that was independent from other known risk factors as residual tumor burden or FIGO stage. ${ }^{6}$ In endometrial carcinoma, two studies postulated a putative role for ezrin in cancer progression. ${ }^{7,10}$ However, to our knowledge, this is the first comprehensive description of ezrin expression in a large set of FIGO I endometrioid carcinomas showing its prognostic value. We found that patients suffering from ezrin-rich endometrioid carcinoma were prone to a poorer prognosis regardless of FIGO grade and pT stage as well as age of patient and with an estimated 2.2 relative risk of death $(P=0.047)$.

Apparently, the cellular localization of ezrin shifted when comparing normal endometrium and endometrial carcinomas from an apical membranous to a predominantly cytoplasmic distribution. Cytoplasmic staining was even accentuated in carcinoma cells loosing their cell-cell contacts. This was observed in both, free floating cells detached from micropapillae or in cells at the tumor-host interface. It is well known that ezrin is exchanged between cytoplasm and membrane. Cytoplasmic ezrin exists in a 'closed' conformation based on intra- or intermolecular interactions between the $\mathrm{N}$ - and Cterminus. Threonine and tyrosine phosphorylation induces an 'open' conformation. In this state ezrin is localized towards the membrane where it modulates F-actin dynamics and tethers the microfilament system to the cytoplasmic face of cell adhesion sites. ${ }^{16}$ Disruption of actin filaments and a decrease in focal adhesion are common features of epithelialmesenchymal transition which is associated with the onset of invasion. Besides a structural role, ezrin may as well act as a signaling or scaffold molecule. Recently, it was revealed that ezrin is involved in modulating signalling pathways acting through Rho $^{17}$ and phosphatidylinositol 3-kinase/Akt. ${ }^{18}$

However, when comparing ezrin levels in endometrial carcinoma with tumor-free endometrium, no straightforward overexpression in carcinomas was detectable. Rather, ezrin expression intensity in normal endometrial epithelium was within the same range as in a subset of carcinomas. Regardless, loss of ezrin expression apparently rendered the tumor less aggressive, as revealed by a better prognosis.

Osteopontin is reported to be an integral part of the CD44-ezrin complex and it is known to associate with poor prognosis of breast and ovarian carcinomas. $^{14,15}$ However, the present study shows no impact on overall survival in endometrial carcinomas. In the contrary, there is a trend that 
strong osteopontin expression correlates with a better prognosis. This finding is supported by a recent study which indicates that osteopontin expression, in contrast to other cancers, was not correlated with tumor stage in endometrial carcinomas. ${ }^{13}$ Like ezrin, osteopontin is not upregulated in endometrial carcinomas compared to normal tissue, and localization of osteopontin was within the cytoplasm of tumor cells. Extracellular osteopontin was of negligible amounts suggesting that stromal cells are not a significant source of osteopontin in endometrial carcinomas as it also has been shown in ovarian carcinomas. ${ }^{15}$ Although immunohistochemical studies could not replace functional investigations, the lack of correlation between ezrin and osteopontin expression suggests that the documented interaction of ezrin and osteopontin ${ }^{11}$ may not be of high importance for the role of ezrin in endometrial carcinoma.

In conclusion, our study revealed a rather high predictive value for tumor-related death of strong ezrin expression in patients with low-stage endometrial carcinomas. This finding, warrants further elucidation in prospective trials to evaluate whether it is useful for therapeutic decisions or not.

\section{Acknowledgements}

We thank Dr Schmidt, Tumor centre, Martin-LutherUniversity, Dr Lantzsch, Elisabeth Hospital Halle, and Dr Wolters, Hospital Merseburg, for providing patients data. The excellent technical assistance of Ms Gulich, Ms Beer and Ms Grosse is gratefully acknowledged.

\section{References}

1 Jemal A, Murray T, Ward E, et al. Cancer statistics. CA Cancer J Clin 2005;55:10-30.

2 Brenner H, Stegmaier C, Ziegler H. Long-term survival of cancer patients in Germany achieved by the beginning of the third millenium. Ann Oncol 2005;16: 981-986.

3 Curto M, McClatchey AI. Ezrin ... a metastatic detERMinant? Cancer Cell 2004;5:113-114.

4 Akisawa N, Nishimori I, Iwamura T, et al. High levels of ezrin expressed by human pancreatic adenocarcinoma cell lines with high metastatic potential. Biochem Biophys Res Commun 1999;258:395-400.
5 Elliott BE, Meens JA, SenGupta SK, et al. The membrane cytoskeletal crosslinker ezrin is required for metastasis of breast carcinoma cells. Breast Cancer Res 2005;7:R365-R373.

6 Köbel M, Gradhand E, Zeng K, et al. Ezrin promotes ovarian carcinoma cell invasion and its retained expression predicts poor prognosis in ovarian carcinoma. Int J Gynecol Pathol 2006, in press.

7 Ohtani K, Sakamoto H, Rutherford T, et al. Ezrin, a membrane-cytoskeletal linking protein, is involved in the process of invasion of endometrial cancer cells. Cancer Lett 1999;147:31-38.

8 Ilmonen S, Vaheri A, Asko-Seljavaara S, et al. Ezrin in primary cutaneous melanoma. Mod Pathol 2005; 18:503-510.

9 Khanna C, Wan X, Bose S, et al. The membranecytoskeleton linker ezrin is necessary for osteosarcoma metastasis. Nat Med 2004;10:182-186.

10 Ohtani K, Sakamoto H, Rutherford T, et al. Ezrin, a membrane-cytoskeletal linking protein, is highly expressed in atypical endometrial hyperplasia and uterine endometrioid adenocarcinoma. Cancer Lett 2002;179:79-86.

11 Zohar R, Suzuki N, Suzuki K, et al. Intracellular osteopontin is an integral component of the CD44ERM complex involved in cell migration. J Cell Physiol 2000;184:118-130.

12 Zhu B, Suzuki K, Goldberg HA, et al. Osteopontin modulates CD44-dependent chemotaxis of peritoneal macrophages through G-protein-coupled receptors: evidence of a role for an intracellular form of osteopontin. J Cell Physiol 2004;198:155-167.

13 Coppola D, Szabo M, Boulware D, et al. Correlation of osteopontin protein expression and pathological stage across a wide variety of tumor histologies. Clin Cancer Res 2004;10:184-190.

14 Rudland PS, Platt-Higgins A, El Tanani M, et al. Prognostic significance of the metastasis-associated protein osteopontin in human breast cancer. Cancer Res 2002;62:3417-3427.

$15 \mathrm{Kim}$ JH, Skates SJ, Uede T, et al. Osteopontin as a potential diagnostic biomarker for ovarian cancer. JAMA 2002;287:1671-1679.

16 Mangeat P, Roy C, Martin M. ERM proteins in cell adhesion and membrane dynamics. Trends Cell Biol 1999;9:187-192.

17 Mackay DJ, Esch F, Furthmayr H, et al. Rho- and racdependent assembly of focal adhesion complexes and actin filaments in permeabilized fibroblasts: an essential role for ezrin/radixin/moesin proteins. J Cell Biol 1997;138:927-938.

18 Gautreau A, Poullet P, Louvard D, et al. Ezrin, a plasma membrane-microfilament linker, signals cell survival through the phosphatidylinositol 3-kinase/Akt pathway. Proc Natl Acad Sci USA 1999;96:7300-7305. 